\title{
Numerical Algorithms for the Analysis of Initial Configuration in a Railway Catenary Structure and Its Code Implementation: A Software Tool CALPE
}

\author{
Jesús Benet, ${ }^{1}$ Fernando Cuartero, ${ }^{2}$ Tomás Rojo, ${ }^{2}$ Pedro Tendero, ${ }^{2}$ and Enrique Arias ${ }^{2}$ \\ ${ }^{1}$ Departamento de Mecánica Aplicada e Ingeniería de Proyectos, Universidad de Castilla-La Mancha, 02071 Albacete, Spain \\ ${ }^{2}$ Departamento de Sistemas Informáticos, Universidad de Castilla-La Mancha, 02071 Albacete, Spain
}

Correspondence should be addressed to Enrique Arias; enrique.arias@uclm.es

Received 9 April 2015; Accepted 2 August 2015

Academic Editor: Evangelos J. Sapountzakis

Copyright (C) 2015 Jesús Benet et al. This is an open access article distributed under the Creative Commons Attribution License, which permits unrestricted use, distribution, and reproduction in any medium, provided the original work is properly cited.

\begin{abstract}
To obtain the best operating conditions in a railway infrastructure, as the pantograph/catenary system, is a priority issue in modern railways, for a correct management and the best performance of this infrastructure. Although most of the published works on this subject are oriented to solving dynamic problems, in order to successfully address these problems a previous static study is necessary. In this paper, we present a methodology for the study of the forces in a railway catenary, according to the equations of statics equilibrium. The results of this work have allowed us to develop two iterative algorithms that calculate a simple and computationally effective lengths of the droppers, which is crucial to determine the initial structural configuration of the catenary parameter. The obtained results have led us to the implementation of a tool to obtain very precise assemblies with significant savings in maintenance, therefore still being used by ADIF, the Spanish railway administration of railways infrastructures.
\end{abstract}

\section{Introduction}

In recent years, railway transportation has experienced considerable progress, yielding ever higher speeds. This requires that there must be adequate conditions in the lines of rail transport. Especially when considering high speeds, it is necessary to maintain a constant flow of electricity on the tensile member for proper operation of railway units. The pantograph/catenary system based on overhead contact line (OCL or catenary) is the most widely used for feeding modern trains. The catenary is a structure formed by cables that supply electricity to the pantograph, which is responsible for obtaining the energy by direct contact through a surface rub, where the development of a rigorous mechanical calculation is essential in order to achieve optimal coupling between both members.

The design of the overhead contact line requires accurate calculations of two of its components: the droppers and tubular brackets. The droppers are the conductors that connect the messenger wire with the contact wires, and they aimed, in general lines, at maintaining contact wires at the same distance with respect to the track. The brackets are the elements that serve to hold the conductors in their position to allow the pantograph taking the energy. Both elements, dropper and brackets, were initially calculated by geometrical methods and in some cases analytically, but in this case with high cost of run time. In order to allow trains to run at higher speed, the calculation of these items with greater accuracy and a reasonable time is required, given the importance of these elements in the correct behavior of trains.

The catenary is a structural system comprising three types of cables, shown in Figure 1: carrier or messenger wire, droppers, and the contact wire, the last one being the cable responsible for supplying electric energy to the pantograph. The catenary is installed in series, called line span, between 15 and 20 spans of about $60 \mathrm{~m}$ each. The messenger and contact wire are mechanically tensioned by a system of pulleys and counterweights at the ends of the series of the line span. In the assembly of the catenary, it is intended that the contact wire acquires a good configuration, 


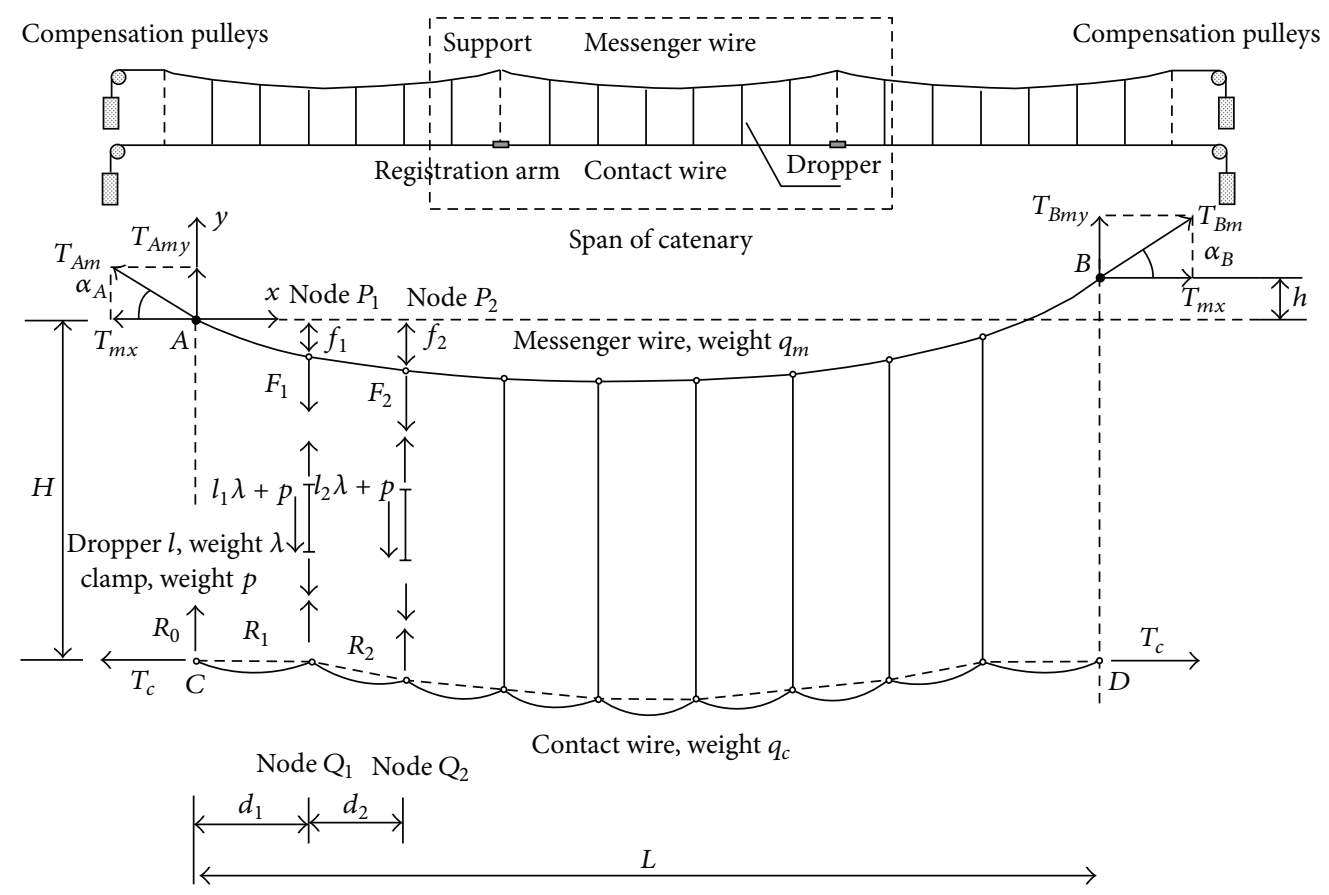

FIGURE 1: Forces in a span of catenary with an irregular geometry.

either parallel to the ground plane, or allowing a certain central deformation (called presag); for that, the droppers, as they are the vertical cables linking the messenger with the contact wire, must have a certain length, which is calculated from a static study of the forces that are in the initial configuration. Yet despite the importance of this problem, it has not been given enough attention in the research, and there are a few numbers of publications in this area. In [1] a method which numerically investigates the behavior of sag and tension in a general catenary structure considering elastic deformation using Finite Element Method (FEM) is presented. Also, an approach using FEM with absolute nodal coordinates is used in [2] in order to obtain the initial configuration and the length of the droppers in a railway catenary. In [3-5] different procedures are presented using FEM, where the cables are modeled as prestressed beams, according to the Euler-Bernoulli equation. In other studies, the static equations of wires are used, whereas in some cases we consider the exact weight of the cable as a uniform load per unit length, see [6], or by approaching such weight as a uniform load per unit of horizontal length (see [4]).

In general, in most of the proposed procedures we need to solve a system of equations. Furthermore, we must take into account that a real assembly line has an irregular geometry with slopes, different heights at the supports (encumbrance), and different settings on the contact wire, depending of the type of span: normal one, overlapping (where one line span is ending, and the other is beginning), switched span (bifurcations), and so forth. Thus, because of all of these considerations, the need to develop a calculation procedure is important, efficient from the computational point of view, and at the same time adapted to any geometry and configuration of the railway line.
To cope with this problem, in this paper we present two methods for studying the problem, by using equations of static wires, and considering the two hypotheses discussed above in this paper: first, it is considered the most accurate scenario where the cable weight is equivalent to a uniform load per unit length, and in the second hypothesis we consider the approximate load, assuming such weight as a uniform load per unit of horizontal length. From the static equations, we develop two iterative algorithms to calculate the forces in the cables and the lengths of the droppers in a railway catenary, in a simple and direct way with minimal computation time, without solving the full system equations, obtaining in both cases very similar results. These results are contrasted with reality, by means of experimental tests, and with other examples published in the literature, obtaining a good numerical agreement.

In order to achieve the speed and accuracy required in the calculations, we have developed a software tool to obtain the length of the droppers and the dimensioning of the brackets, implementing the second one of the two proposed methods indicated previously. We have chosen this method because it has been observed that the difference between both methods is low, on the order of a hundredth of a millimeter, and also, this method is simpler and more efficient than the first one. The program performs calculations for different types of spans and for a full set of a sequence of spans (line span), obtaining the length of the droppers and the section and length of the brackets needed.

The computer tool manages a database system created to store, identify, and manage the large amount of information about the large number of components and parts that constitute a span or a line span. Also, it is structured in their different components: user interface, calculation algorithms, 


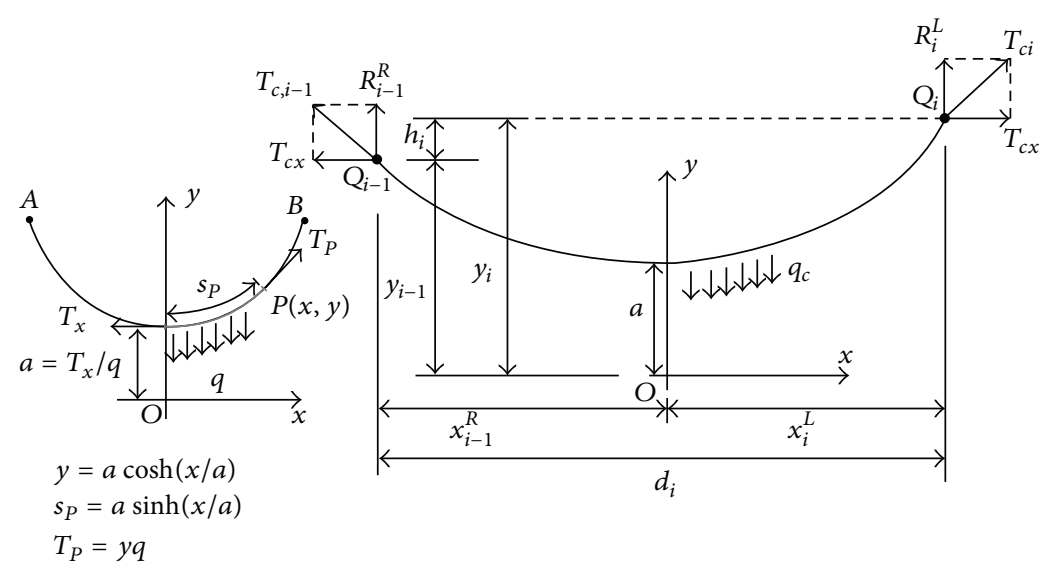

FIGURE 2: Catenary equation and segment of contact wire between droppers.

and generation of several graphics. The results obtained are also presented graphically by plotting, in a scale, each of the spans calculated, indicating the values and the position of each of the droppers and brackets, attached to the initial values, in a complete report useful to an engineer for the correct installation of the catenary.

The paper is structured as follows: in Section 2 the study of the problem takes place regarding the first hypothesis; in Section 3 the study is performed with the second hypothesis; in Section 4 the results of both hypotheses are contrasted and compared in turn with other examples published in the scientific literature; in Section 5 the software tool with all its benefits are described; finally in Section 6 the findings are discussed.

\section{Calculation Considering the Weight of the Wire as a Uniform Load per Unit of Length}

2.1. Equation of the Wire Segment between 2 Points. In our study, we begin from the equations of a static wire, according to [7]. Let us assume a segment wire $A B$ with a weight per unit of length $q$, where $P(x, y)$ represents a point, and the equation of the curve that forms this wire may be expressed in the system of axes $O x y$ in Figure 2, as follows:

$$
\begin{aligned}
& y=a \cdot \cosh \frac{x}{a}, \\
& a=\frac{T_{x}}{q} .
\end{aligned}
$$

This equation is known in the literature as catenary, and the constant " $a$ " is known as the parameter of the catenary. The horizontal component of the mechanical force at any point $T_{x}$ is constant and equals the horizontal component of the support reactions $A$ and $B$. The mechanical force at a point $P(x, y)$ may be expressed as

$$
T_{p}=q \cdot y \text {. }
$$

And the length of the arc between the minimum and the point $P$ is

$$
s_{p}=a \cdot \sinh \frac{x}{a}
$$

2.2. Reaction of the Droppers. The first problem to be considered is the calculation of the reactions or forces in the droppers of each span, these reactions are caused by the weight of the contact wire underpinning, and its calculation can be performed from the reactions at the supports of a segment of the contact wire, located between points $Q_{i-1}$ and $Q_{i}$, representing the lower ends of two adjacent droppers, according to Figures 1 and 2 , with $i=1, \ldots, n$, where $n$ is the number of droppers in the span.

As the contact wire has a predefined configuration, we know the relative positions between $Q_{i-1}$ and $Q_{i}$, given by the horizontal distance between droppers $d_{i}$ and by their height difference $h_{i}$, as it is shown in Figure 2. Also, admitting that the contact wire is tightened at the ends of the line span, by counterweights and compensation pulleys, being vertically supported by the droppers, and that this configuration is nearly horizontal, we can assume the hypothesis that the horizontal component of the tension of the contact wire, represented by $T_{c x}$, is constant across the line, and almost the same to the force caused by the counterweights, represented by $T_{c}$. Furthermore, we represent the weight wire per unit length as $q_{c}$, and the constant " $a$ " for the contact wire will be $a=T_{c} / q_{c}$. Therefore, according to the equation of the curve of the cable:

$$
\begin{aligned}
& h_{i}=y_{i}-y_{i-1}=a \cdot \cosh \left(\frac{x_{i}^{L}}{a}\right)-a \cdot \cosh \left(\frac{d_{i}-x_{i}^{L}}{a}\right) \\
&=a \cdot \frac{e^{x_{i}^{L} / a}+e^{-x_{i}^{L} / a}}{2}-a \cdot \frac{e^{\left(d_{i}-x_{i}^{L}\right) / a}+e^{-\left(d_{i}-x_{i}^{L}\right) / a}}{2} \\
&=\frac{a \cdot e^{x_{i}^{L} / a}}{2}\left(1+e^{-2 x_{i}^{L} / a}-e^{d_{i} / a} \cdot e^{-2 x_{i}^{L} / a}-e^{-d_{i} / a}\right), \\
& i=1, \ldots, n .
\end{aligned}
$$


If $b_{i}=e^{d_{i} / a}, z_{i}=e^{x_{i}^{L} / a}$, we have

$$
\begin{aligned}
& h_{i}=\frac{a \cdot z_{i}}{2}\left(1+\frac{1}{z_{i}^{2}}-\frac{b_{i}}{z_{i}^{2}}-\frac{1}{b_{i}}\right), \\
& z_{i}^{2}\left(a-\frac{a}{b_{i}}\right)-2 h_{i} z_{i}+\left(a-a b_{i}\right)=0,
\end{aligned}
$$

$$
i=1, \ldots, n
$$

and here,

$$
\begin{aligned}
z_{i} & =\frac{2 h_{i} \pm \sqrt{4 h_{i}^{2}-4 a^{2}\left(1-1 / b_{i}\right)\left(1-b_{i}\right)}}{2 a\left(1-1 / b_{i}\right)}, \\
x_{i}^{L} & =a \cdot \log z_{i}, \\
x_{i-1}^{R} & =d_{i}-x_{i}^{I},
\end{aligned}
$$

$$
i=1, \ldots, n,
$$

obtaining a single valid solution for $z_{i}>0$, corresponding to the positive sign of the radical. The vertical reaction in $Q_{i}$ due to the segment $Q_{i-1}-Q_{i}$, located on the left of $Q_{i}$, will be:

$$
R_{i}^{L}=q_{c} a \cdot \sinh \frac{x_{i}^{L}}{a}, \quad i=1, \ldots, n .
$$

To obtain the total reaction $Q_{i}$ we need also to consider the effect of segment $Q_{i}-Q_{i+1}$ located on the right, resulting in

$$
\begin{aligned}
& R_{i}=R_{i}^{L}+R_{i}^{R}=q_{c} a \cdot \sinh \frac{x_{i}^{L}}{a}+q_{c} a \cdot \sinh \frac{x_{i}^{R}}{a}, \\
& i=1, \ldots, n,
\end{aligned}
$$

where $x_{i}^{R}$ is calculated similar to $x_{i-1}^{R}$ but particularizing for the segment $Q_{i}-Q_{i+1}$.

2.3. Deformation on the Messenger. In order to calculate the length of the droppers, we need to determine the configuration of the equilibrium of the messenger at each span, and from here, and assuming that the configuration of the contact wire is known, then the calculation of the lengths of the droppers is immediate. In a catenary span, the messenger is subjected to the vertical load corresponding to its own weight, which in this case is a uniform load per unit of length $q_{m}$, and the reactions of the droppers, represented by forces $R_{i}, i=1, \ldots, n$, which are calculated according to (4)(8). However, these reactions must be corrected taking into account the constant weight of clamps, and the own weight of the droppers, resulting for a generic dropper $i$, and the overall reaction is

$$
F_{i}=R_{i}+p+l_{i} A \gamma, \quad i=1, \ldots, n,
$$

where $F_{i}$ represents the overall reaction corrected in the dropper $i, l_{i}$ its length, $p$ the weight of the holding elements, $A$ the area of the section of the dropper, and $\gamma$ the density of the material.
According to (1), the equation of a cable under the action of its own weight is entirely defined by the parameter " $a$," which depends on the weight of the wire, which is known, and the horizontal force, which is initially ignored. The messenger of a catenary span may be considered as formed by a series of discontinuous arcs of the same curve, as it is shown in Figure 3 where $P_{i}, i=1, \ldots, n$, are the nodes or junction points of the arcs representing the upper grips of the droppers.

By locating such points on the theoretical curve, we split them into two different points $P_{i}^{L}$ and $P_{i}^{R}$ with different mechanical tension, as a discontinuity appears in the force, due to the vertical downward force of the dropper $F_{i}$. This force changes the vertical component of the cable tension, with the horizontal tension $T_{m x}$ remaining constant throughout the span.

Contrary to the contact wire, the messenger wire is fixed at supports $A$ and $B$, varying the horizontal tension $T_{m x}$ from one span to another along the line span. Thus, given the weight of the cable $q_{m}$, if we know the components of the reaction of the wire at the left mast $A$, and the forces in the droppers $F_{i}$ are also known, it is possible to calculate immediately the cable configuration. The abscissa of support $A$ in the reference system $O x y$ of the theoretical curve in Figure 3 is

$$
x_{A}=a \cdot \cosh ^{-1}\left(\frac{y_{A}}{a}\right)=\frac{T_{m x}}{q_{m}} \cosh ^{-1}\left(\frac{T_{A m}}{T_{m x}}\right),
$$

where $y_{A}=T_{A m} / q_{m}$ and $a=T_{m x} / q_{m}$, being $T_{A m}$ the total mechanical tension on $A$. Thus, the position $P_{1}^{L}$, corresponding to the node where the first dropper is gripped in the reference system of Figure 3, will be

$$
\begin{aligned}
& x_{1}^{L}=x_{A}-d_{1}, \\
& y_{1}^{L}=\frac{T_{m x}}{q_{m}} \cosh \left(\frac{x_{1}^{L} q_{m}}{T_{m x}}\right) .
\end{aligned}
$$

The vertical position of node $P_{1}$, with respect to the left support $A$, is

$$
h_{1}=y_{1}^{L}-y_{A} .
$$

The vertical force in $P_{1}^{L}$ is

$$
T_{1 y}^{L}=\sqrt{\left(T_{1}^{L}\right)^{2}-T_{m x}^{2}}=\sqrt{\left(q_{m} y_{1}^{L}\right)^{2}-T_{m x}^{2}}
$$

And vertical force and total mechanical tension in $P_{1}^{R}$ are

$$
\begin{aligned}
& T_{1 y}^{R}=T_{1 y}^{L}-F_{1}, \\
& T_{1}^{R}=\sqrt{\left(T_{1 y}^{R}\right)^{2}+T_{m x}^{2}}
\end{aligned}
$$

These equations may be extended to the different attachment points $P_{i}$, with $i=1, \ldots, n+1$, of the droppers with the messenger wire, including the right support. Thus, for a 


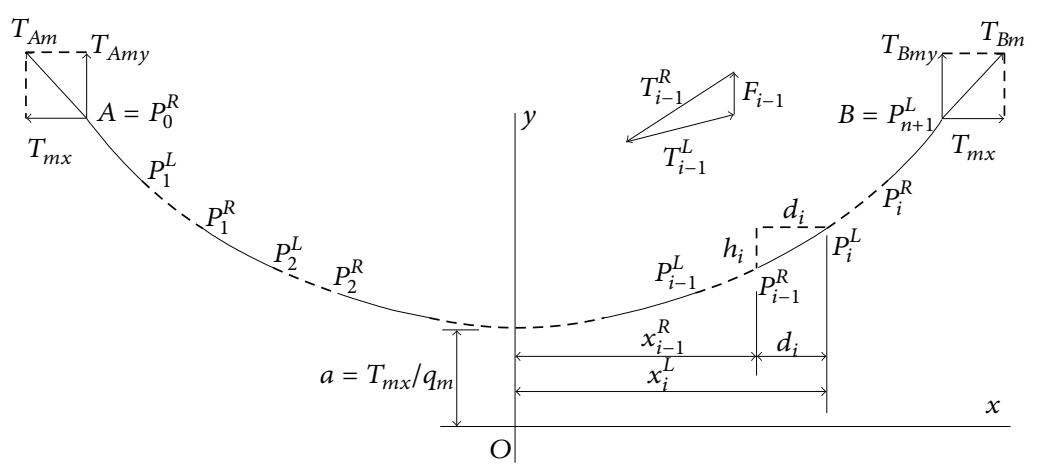

Figure 3: Configuration of the messenger wire in a catenary span.

generic cable segment between $P_{i-1}$ and $P_{i}$, the position of the point $P_{i-1}^{R}$ in the reference system of Figure 3 will be

$$
\begin{aligned}
x_{i-1}^{R} & =\frac{T_{m x}}{q_{m}} \cosh ^{-1}\left(\frac{T_{i-1}^{R}}{T_{m x}}\right), \\
y_{i-1}^{R} & =\frac{T_{i-1}^{R}}{q_{m}},
\end{aligned}
$$

$$
i=1, \ldots, n+1 \text {. }
$$

For position $P_{i}^{L}$ is

$$
\begin{aligned}
& x_{i}^{L}=x_{i-1}^{R} \pm d_{i}, \\
& y_{i}^{L}=\frac{T_{m x}}{q_{m}} \cosh \left(\frac{x_{i}^{L} q_{m}}{T_{m x}}\right),
\end{aligned}
$$

$$
i=1, \ldots, n+1,
$$

where the sign in expression $d_{i}$ depends on whether $P_{i-1}^{R}$ is on the right or left of the minimum point of the theoretical curve. The vertical position of $P_{i}$ with respect to $P_{i-1}$ in the real curve is

$$
h_{i}=y_{i}^{L}-y_{i-1}^{R}, \quad i=1, \ldots, n+1 .
$$

This equation allows us to obtain the vertical position of $P_{i}$ in the Coordinates System Axy of Figure 1, corresponding to the upper end of the dropper. The lower end $Q_{i}$ is defined by the configuration imposed on the contact wire, being the length of the dropper $l_{i}$ as the vertical distance $P_{i} Q_{i}$, for $i=1, \ldots, n$. The process continues with the calculation of the vertical tension in $P_{i}^{L}$ :

$$
\begin{aligned}
T_{i y}^{L}=\sqrt{\left(T_{i}^{L}\right)^{2}-T_{m x}^{2}}=\sqrt{\left(q_{m} y_{i}^{L}\right)^{2}-T_{m x}^{2}} & \\
& \\
& i=1, \ldots, n+1 .
\end{aligned}
$$

Vertical tension and total stress in $P_{i}^{L}$ are

$$
\begin{aligned}
& T_{i y}^{R}=T_{i y}^{L} \pm F_{i}, \\
& T_{i}^{R}=\sqrt{\left(T_{i y}^{R}\right)^{2}+T_{m x}^{2}}, \\
& i=1, \ldots, n .
\end{aligned}
$$

When, again, the sign of $F_{i}$ depends on whether $P_{i}^{L}$ is in the right or the left of the minimum point of the theoretical curve, repeating the cycle for the next droppers using (15)-(19).

2.4. Initial Estimation of the Horizontal Tension of the Messenger. The above equations allow us to calculate successively the deformations of the messenger wire in the points $P_{i}$, $i=1, \ldots, n+1$, for a given span, and from here, to calculate the corresponding length of the droppers. However, to begin the procedure we need to know, furthermore, the components of the reactions of the messenger on the left support $A: T_{A y}, T_{m x}$. Although these reactions are unknown, it is assumed that we know the total tension in the cable in the support $A$, represented by $T_{A}$, which in the first span is equal to the tension produced by the counterweight of the pulley, from this data it is possible to obtain a first approximation of the horizontal component $T_{m x}$, assuming in this case that the cable is subjected to a uniform horizontal load per unit of length. Thus, if $L$ represents the length of the span, $h$ is the height difference between supports $A$ and $B$, according to Figure 1 , and $M_{B}$ is the moment of the weight of the cable and the reactions $F_{i}$ of the droppers with respect to the right support $B$, then, the moment equilibrium equation of the forces acting onto the messenger with respect to $B$, together with the vectorial equation of the absolute tension of the cable in $A$, is

$$
\begin{aligned}
T_{A m y} L+T_{m x} h-M_{B} & =0, \\
T_{A} & =\sqrt{T_{m x}^{2}+T_{A m y}^{2}} .
\end{aligned}
$$

With the above two equations we have a system with two unknowns corresponding to the components of the reaction at $A$, and these equations can be solved to calculate $T_{m x}$ and $T_{A m y}$ :

$$
\begin{aligned}
T_{m x} & =\frac{2 M h \pm \sqrt{4 M^{2} h^{2}-4\left(h^{2}+L^{2}\right)\left(M^{2}-L^{2} T_{A}^{2}\right)}}{2\left(h^{2}+L^{2}\right)}, \\
T_{A m y} & =\sqrt{T_{A}^{2}-T_{m x}^{2}} .
\end{aligned}
$$

For typical values in a system, there exists a single valid solution for $T_{m x}>0$, corresponding to the positive sign 
of the radical. With this approximate value for $T_{m x}$ we can calculate the deformations on the messenger, the corresponding lengths of the droppers, and mechanical forces, from (4) to (19). The approximate value of $T_{m x}$ may be corrected at the end of the calculation by comparing the actual known position of the right support $B$ with respect to the left $A$, that is, $h$ with the calculated $h^{\prime}$ from the estimated value of $T_{m x}$, and the difference between the values $h$ and $h^{\prime}$ is a measurement of the error produced in the calculation process, if this difference is under a certain allowable error value, $T_{m x}$, and therefore the calculated deformation of the messenger and the lengths of the droppers are correct; otherwise, we need additional calculations for a new improved value $T_{m x}$. This new value is obtained, again, from a revised estimated value of cable tension in the right support $T_{B}$, according to the error in the calculation of the position of $B$ given by $h^{\prime}-h$. If $\varepsilon$ is the allowed error on the calculated position $B$, the algorithm for improved $T_{B}$ and $T_{m x}$ values will be

$$
\begin{array}{ll}
\text { if } & \left|h^{\prime}-h\right| \leq \varepsilon \\
\text { then } & \text { STOP } \\
\text { else } & T_{B}=q_{m}\left[y_{B}-\left(h^{\prime}-h\right)\right] \\
& T_{m x}=\sqrt{T_{B}^{2}-T_{B y}^{2}},
\end{array}
$$

where $T_{B y}$ is equivalent to $T_{n+1, y}^{L}$ in (18) and $y_{B}$ to $y_{n+1}^{L}$ in (16), repeating the calculations until the calculated error for the position of $B$ is under the permissible error $\varepsilon$. We need to take into account that initially the lengths and the corresponding weights of the droppers are unknown; thus, in principle we assume these values are equal to zero, and they are corrected for each iteration. Once we have calculated the values for a span, we proceed to study the following one to the right, taking the absolute tension on the left support of the new span as the calculated tension in the right support of the previous one, proceeding in the same way for the full line span from left to right, beginning with the span located first to the left. In the calculation of the first span, the mechanical tension on the left support is the force given by the counterweight pulley.

2.5. Algorithm to Calculate the Lengths of Droppers. The algorithm for calculating the droppers throughout a line span is as follows.

(1) The calculation will be done span by span from left to right. Initially, the lengths and weights of the droppers of each span will be assumed zero. The total mechanical tension in the left support of the messenger is assumed to be known for the first span and is given by the counterweight of the compensating pulley.

(2) We calculate the reactions in the droppers of the span according to the configuration imposed on the contact wire, defined in (4)-(8).

(3) We obtain a first estimation of the reactions on the left support of the messenger, assuming a uniform load per unit of horizontal length, according to (21).
(4) We calculate the new configuration, mechanical tensions of the messenger, and the length of the droppers, in accordance with (9)-(19).

(5) Check the error produced in the calculation of the span according to (22). If this error is under the permissible error $\varepsilon$, the calculation is correct and we pass to calculate the next span, going back to point 1 . Otherwise, we recalculate the value of the horizontal tension in the messenger $T_{m x}$ according to (22) and the reactions of the droppers for the last value of their lengths, according to (9), returning to step (4) until the error in the calculation of the span is under the permissible error $\varepsilon$.

\section{Calculation Considering the Weight of the Wire as a Uniform Load per Unit Horizontal Length}

3.1. Calculating Dropper Reactions. As in the previous hypothesis, the calculation will be done span by span, beginning from the left. When we assume that the weight of the cable is as a uniform horizontal length per unit of load, the calculation of the dropper reactions is immediate. According to Figure 4, if we consider the moment equilibrium in the segment of the contact wire located between $Q_{i-1}$ and $Q_{i}$ with respect to $Q_{i-1}$, we get in an immediate way the reaction in $Q_{i}$, say $R_{i}^{L}$; thus, repeating the process for segments $Q_{i}$ to $Q_{i+1}$ we obtain $R_{i}^{R}$, and the global reaction in node $Q_{i}$ is the sum of the above two reactions. As in the previous hypothesis, we assume that the horizontal tension of the contact wire $T_{c x}$ remains constant throughout the line, and it is equal to $T_{c}$ the tension produced by the counterweight pulley on the end of the line span, resulting in

$$
\begin{array}{r}
R_{i}=R_{i}^{L}+R_{i}^{R}=\frac{1}{2}\left(d_{i}+d_{i+1}\right) q_{c}+T_{c}\left(\frac{h_{i}}{d_{i}}+\frac{h_{i+1}}{d_{i+1}}\right) \\
i=1, \ldots, n .
\end{array}
$$

3.2. Calculation of Droppers Length and Deformations. Once we have calculated the reactions on the droppers due to the weight of the contact wire, the overall reaction is calculated by adding the previous value of the weight of the dropper and the fixed weight of the clamps, according to (9). From these reactions and the absolute mechanical tension on the left support of the messenger, we calculate the components of the reactions in the left support, according to (21). In this case the assumption made in (21) to the wire weight coincides with the assumed hypothesis for the full calculation of the span. Next, we determine the configuration of the messenger by considering the moment equilibrium of the different segments of wire between the left support $A$, and the different nodes or grip points for droppers, $P_{i}$, for $i=1, \ldots, n$, according to Figure 5, resulting in

$$
\sum M_{C}=0
$$




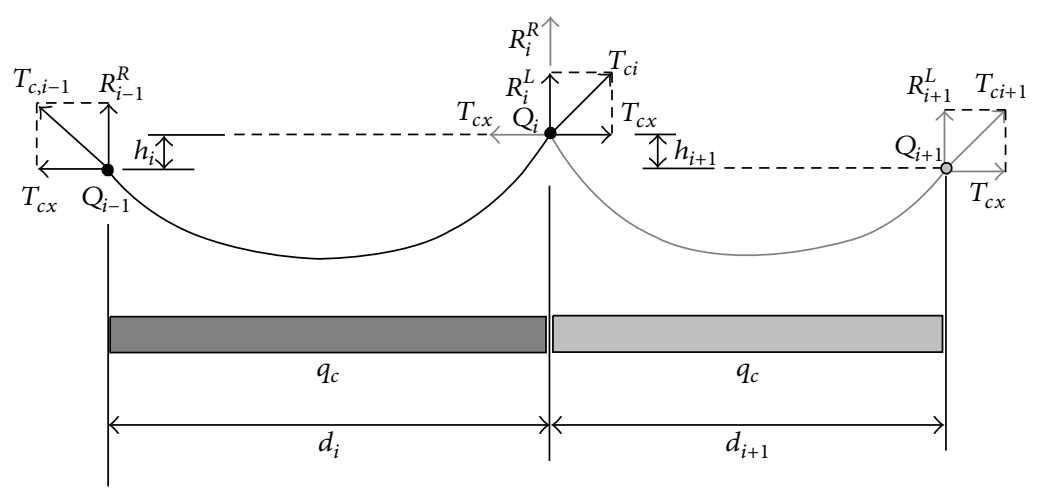

FIGURE 4: Segments of the contact wire. Reactions on the droppers.

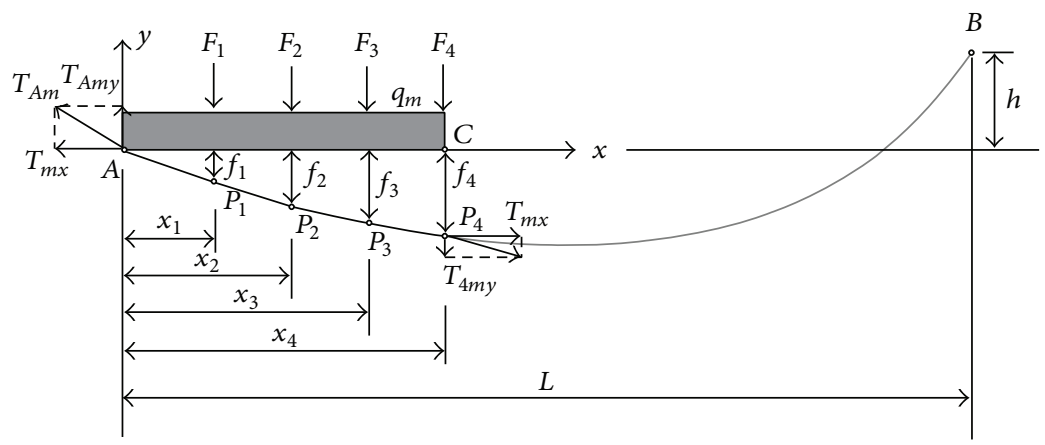

FIGURE 5: Equilibrium in a segment of messenger wire.

$$
\begin{array}{r}
f_{i}=\frac{1}{T_{m x}}\left[T_{A m y} x_{i}-q_{m} \frac{x_{i}^{2}}{2}-\sum_{j=1}^{i} F_{j}\left(x_{i}-x_{j}\right)\right], \\
i=1, \ldots, n .
\end{array}
$$

Finally, once we know the deformations of the messenger, it is possible to calculate the lengths of the droppers by an iterative process, similar to that used in the previous hypothesis. In this case the comparison value for each step is taken from the relative change of the horizontal tension $T_{m x}$. In each iteration this value is only adjusted for the weight of the droppers, corresponding to the last estimation of their lengths, so if $T_{m x}$ is the last calculated value of the horizontal tension, $T_{m x}^{\prime}$ is the previous value, and $\varepsilon$ is the admissible error; then the calculation will be completed when the span is fulfilling:

$$
\left|\frac{T_{m x}-T_{m x}^{\prime}}{T_{m x}}\right|<\varepsilon .
$$

\section{Comparative with Related Work}

4.1. First Comparative. Although there are few published data on static calculations of catenary, because main works are focused on dynamic studies, our results have been compared to other examples published in the literature with those obtained by applying the two proposed algorithms in this
TABLE 1: Parameters example reference [8].

\begin{tabular}{lc}
\hline Span length $L(\mathrm{~m})$ & 20 \\
Encumbrance $H(\mathrm{~m})$ & 1 \\
Number of droppers & 2 \\
Distance of droppers to left support $(\mathrm{m})$ & $5.5,14.5$ \\
Droppers, mass/unit length $A \gamma(\mathrm{kg} / \mathrm{m})$ & 0.14 \\
Mass clamps $p(\mathrm{~kg})$ & 0.25 \\
Contact wire, mass/unit length $q_{c}(\mathrm{~kg} / \mathrm{m})$ & 1.068 \\
Contact wire, mechanical tension $T_{c}(\mathrm{KN})$ & 15 \\
Contact wire flexural rigidity $E I_{c}\left(\mathrm{Nm}^{2}\right)$ & 150 \\
Messenger wire, mass/unit length $q_{m}(\mathrm{~kg} / \mathrm{m})$ & 0.6 \\
Messenger wire, mechanical tension $T_{m}(\mathrm{KN})$ & 15 \\
\hline
\end{tabular}

paper. First we have considered [8], in which the characteristics of a catenary with a single span with two droppers is studied for the verification of dynamic problems. Although the method of calculation used is not fully explained, the complete characteristics of the catenary and the lengths of the droppers are provided. This same example is also studied in [6], in which the equations of statics are also used considering the weight of the cable as a uniform load per unit of length; in this case the length of the droppers is obtained by a different method from that proposed in this paper, by solving a nonlinear system of equations. The parameters of the catenary can be seen in Table 1 . 
TABLE 2: Results of first comparative.

\begin{tabular}{lcccc}
\hline & $\begin{array}{c}\text { Arnold and } \\
\text { Simeon [8] }\end{array}$ & $\begin{array}{c}\text { López-Garcia } \\
\text { et al. [6] }\end{array}$ & Method 1 & Method 2 \\
\hline $\begin{array}{l}\text { Length } \\
\text { dropper (m) }\end{array}$ & 0.95 & 0.9579 & 0.95512 & 0.95512 \\
\hline
\end{tabular}

TABLE 3: Parameters example reference [4].

\begin{tabular}{lc}
\hline Span length $L(\mathrm{~m})$ & 50 \\
Encumbrance $H(\mathrm{~m})$ & 0.96 \\
Number of droppers & 10 \\
& $2.5,7.5,12.5$, \\
Distance of droppers to left support $(\mathrm{m})$ & $17.5,22.5$, \\
& $27.5,32.5,37.5$, \\
Droppers, mass including clamps $(\mathrm{kg})$ & $42.5,47.5$ \\
Dropper stiffness $(\mathrm{N} / \mathrm{m})$ & 0.4 \\
Contact wire, mass/unit length $q_{c}(\mathrm{~kg} / \mathrm{m})$ & $10^{5}$ \\
Contact wire, mechanical tension $T_{c}(\mathrm{KN})$ & 0.987 \\
Contact wire flexural rigidity $E I_{c}\left(\mathrm{Nm}{ }^{2}\right)$ & 12 \\
Messenger wire, mass/unit length $q_{m}(\mathrm{~kg} / \mathrm{m})$ & 130 \\
Messenger wire, mechanical tension $T_{m}(\mathrm{KN})$ & 0.605 \\
Messenger wire, flexural rigidity $E I_{m}\left(\mathrm{Nm}{ }^{2}\right)$ & 12 \\
\hline
\end{tabular}

The obtained results for the lengths of the droppers are shown in Table 2. Whereas the span is symmetrical and the two droppers are equal, we indicate only a single result. In the second column of the table we shown the value of the length obtained in [8], and in the third column the same value obtained by the method of reference [6]. We can see that there is a difference of $0.83 \%$ between them which is not significant, even more because we do not know the rounding method. In the last two columns we display the results obtained in accordance with the two methods proposed in the this work. We can see a good agreement in all cases but for the two methods proposed in this paper it is absolute coincidence, although the equations are completely different. The results obtained using the proposed methods also have been expressed with five decimal places in order to better appreciate the possible differences.

4.2. Second Comparative. Another comparative has been with the example proposed in [4], in this case we have a type of catenary used in Korean railways. The used parameters are shown in Table 3. As we can see in that table, in this case there is a span of $50 \mathrm{~m}$. with 10 droppers. Since the span is symmetrical, the shown results in Tables 4 and 5 refer only to the first five droppers. Furthermore, in this example we have considered two cases: a first case with no sag in the contact wire and a second one with $5 \mathrm{~cm}$ of sag. Although in [4] the features of the registration arm are shown we have found, according to the results of the example, that its effect is not considered in the calculation; that is, it is supposed in that example that the arm exerts no upward force on the contact wire. The authors proposed also two methods, a first one, by applying the equations of static wire, and a second by the finite element method. Furthermore, as it is shown in the parameters in Table 3 , the real weight of the droppers, of which it depends on their length, is not considered, but a fixed value of $0.4 \mathrm{~kg}$ is taken, including in such value the fixed weights of the clamps. The results of these two methods are compared with those obtained by our proposal. As in the preceding case, these results are shown with five decimal places to better compare the differences.

As we can see in the tables, the results are generally quite consistent in all methods. First, if we compare the results of methods 1 and 2 of our work (last two rows of Tables 4 and 5) values are virtually identical. Thus, we can consider that the error made when approximating the weight of wires as a uniform load per unit of horizontal length is approximately one hundredth of a millimeter, which is negligible. The largest differences appear when comparing the results of the methods developed using the equations of static equilibrium for cables, as is the case of the first method of [4] and both methods of this paper, with respect to the second method of the same reference, which employs the finite elements method. In this last case, it has been considered the bending stiffness by the Euler-Bernoulli equation, with a higher computational cost, because it is necessary to solve a system of equations. However, the differences are still very small, in the order of a millimeter, and thus, we think that it is not interesting due to the higher cost in time.

Finally, we have studied the convergence of the algorithms for our two methods proposed for this last example, assuming the permissible error estimated to be in $\varepsilon=10^{-8}$, and the results are shown in Figure 6 in the form of graphics in which the abscissa represents the number of iterations and the ordinate error in each iteration. The first graph on the left corresponds to the first method, where we can see a fast convergence, being required nine iterations. For the second method, we have not shown the graph as the convergence in this case is straightforward. The explanation for this is due to the fact that, in the first method, the horizontal stress of the supporting $T_{m x}$ is corrected in each iteration taking into account the error in the initial hypothesis, to consider the approximate weight of the cable, given by (21) and the correction by the own weight of the droppers to be modified according to its length, being this one initially unknown; while for the second method the correction is effected only by the weight of the droppers, as in the example from [4] this weight directly adopts a constant value corresponding to a fixed weight (clamps) of $0.4 \mathrm{~kg}$, thus, the results are obtained directly. In order to better study the influence of the actual weight of the droppers in the error, we have repeated this last example but assuming droppers of $16 \mathrm{~mm}^{2}$ and copper material density $8,900 \mathrm{~kg} / \mathrm{m}^{3}$, besides keeping the fixed weight of $0.4 \mathrm{~kg}$ for the clamping elements, and the results for the proposed two methods are shown in the second and third graphics of Figure 6. In both cases, a rapid convergence with almost identical results are achieved, although the first method needs thirteen iterations while the second method needs only four iterations. 

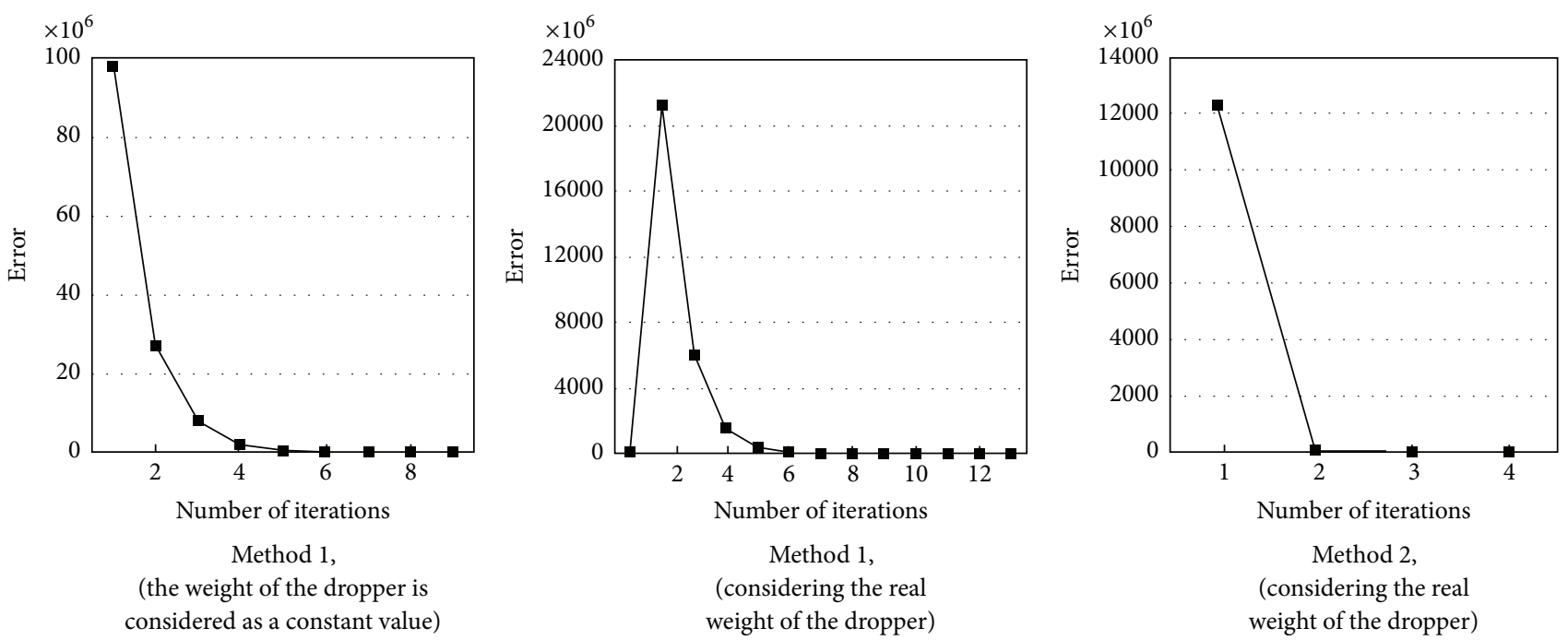

Figure 6: Comparison of methods.

TABLE 4: Length of the droppers and comparison results, reference [4], without presage.

\begin{tabular}{|c|c|c|c|c|c|}
\hline & $\begin{array}{c}\text { Dropper } 1 \\
(\mathrm{~m})\end{array}$ & $\begin{array}{c}\text { Dropper } 2 \\
(\mathrm{~m})\end{array}$ & $\begin{array}{c}\text { Dropper } 3 \\
(\mathrm{~m})\end{array}$ & $\begin{array}{c}\text { Dropper } 4 \\
(\mathrm{~m})\end{array}$ & $\begin{array}{c}\text { Dropper } 5 \\
(\mathrm{~m})\end{array}$ \\
\hline Cho et al. [4], method 1 & 0.876 & 0.740 & 0.637 & 0.569 & 0.535 \\
\hline Cho et al. [4], method 2 & 0.875 & 0.738 & 0.636 & 0.567 & 0.533 \\
\hline Present work, method 1 & 0.87606 & 0.73929 & 0.63671 & 0.56833 & 0.53414 \\
\hline Present work, method 2 & 0.87607 & 0.73930 & 0.63673 & 0.56834 & 0.53415 \\
\hline
\end{tabular}

TABLE 5: Length of the droppers and comparison results, reference [4], with presage.

\begin{tabular}{lccccc}
\hline & $\begin{array}{c}\text { Dropper 1 } \\
(\mathrm{m})\end{array}$ & $\begin{array}{c}\text { Dropper 2 } \\
(\mathrm{m})\end{array}$ & $\begin{array}{c}\text { Dropper 3 } \\
(\mathrm{m})\end{array}$ & \multicolumn{2}{c}{$\begin{array}{c}\text { Dropper 4 } \\
(\mathrm{m})\end{array}$} \\
\hline Cho et al. [4], method 1 & 0.876 & 0.779 & 0.706 & 0.658 & 0.633 \\
Cho et al. [4], method 2 & 0.875 & 0.778 & 0.705 & 0.657 & 0.633 \\
Present work, method 1 & 0.87606 & 0.77881 & 0.70587 & 0.65725 & 0.63294 \\
Present work, method 2 & 0.87607 & 0.77882 & 0.70588 & 0.65725 & 0.63295 \\
\hline
\end{tabular}

Thus, we can conclude that the different methods used to compute the results of the catenary forces offer no significant differences. But, on the contrary, by using either an algorithm based on finite elements method or a system of equations, a greater complexity is introduced in computing time, which is an important handicap in order to obtain a computer tool suitable for engineers. In particular, our second method, theoretically more approximated, obtains the same results with a minor number of iterations, and, consequently, computing time. Moreover, these kinds of approaches, used in other methodologies, make the addition of some other considerations difficult, such as different geometries, different heights at the masts, curves, and auxiliary elements such as cantilever.

\section{Computer Tool CALPE}

The algorithms here presented have been developed by the cooperation between CALPE team of the University of Castilla-La Mancha, and Adif, the Administrator of Railway Infrastructures, which is a state-owned company that answers to the Ministerio de Fomento. Adif plays a leading role in promoting the railway sector, working towards converting it into the ideal mode of transport and facilitating access to the infrastructure under fair conditions. This tool is an example of cooperation between the industry and the Academia.

The implemented tool has been called CALPE (Spanish acronym for CáLculo de Péndolas), and, according to the engineers of Adif, by using it together with InDiCa tool, the cost of the infrastructure can be reduced dramatically. 
In its own magazine [9] Adif indicates that the company saves about one million euros per $100 \mathrm{~km}$ installed, on the basis of its own estimations. Moreover, these software tools are subject to national patents developed jointly between UCLM and Adif and are transferred to this company for its commercial exploitation.

In the first part of this paper, a mathematical model of the problem has been developed and it has been solved, but this model and the solution have to be translated in a numerically effective method. This method has been described in Section 2.5. To carry out the implementation in a real platform it is necessary to consider that this software tool is used in the industry and in the real life by civil engineers. So, this software has to accomplish a set of features to be considered as a high-quality mathematical software [10].

(1) Power and Flexibility. The developed algorithms can be used to solve several different variations of the original problem and the closely associated problems.

(2) Easily to Read and Modify. The software should be well documented. Then, the documentation should be clear and easy to read, even for nontechnical users.

(3) Portability. The implemented algorithms should be able to run on different computers with few or no changes.

(4) Robustness. It should be able to deal with unexpected situations during execution.

(5) Based on a Numerically Effective Algorithm. It should be based on an algorithm that has attractive numerical properties.

All the previous features have been taken into account during the development of CALPE tool. In particular, those good features have been achieved as follows.

(1) Power and Flexibility. Both are achieved by means of dealing with different configuration of the same problem: considering wind effect or not, from 1 to 4 pantographs, normal or stitched catenary, and so forth.

(2) Easily to Read and Modify. CALPE project is a continuous work during near 20 years that started with the first version of CALPE application. Since this first version, and taking into account that a lot of persons have been working on the project, readability has been ever a main objective in the code of the application.

(3) Portability. The source and base code of different versions of CALPE have been successfully tested in different platforms and different versions operating system Windows with no or little changes.

(4) Robustness. A good error treatment allows us to deal with anomalous situations, alerting when and where they are produced.

(5) Based on a Numerically Effective Algorithm. As we have presented in Section 2.5. This effectiveness is shown considering two parameters. (a) Efficiency in terms of Execution Time. The efficiency of an algorithm is measured by the amount of computer time consumed in its implementation. In order to achieve with this requirement we have selected the easier method to solve the problem when no advantages have been considered.

5.1. Related Applications. Previously to present our tool, we introduce some related work that it is found in the literature. In fact, there are no references about tools implementing the calculation of droppers. But this is part of a major task, such as the interaction of pantograph and catenary, where this calculation plays an important role. This occurs also with our tool InDiCa, presented in [3], where an important part is this calculation that we present in this work. Another work, also more related to the dynamic interaction, is as follows.

(i) The PANDA tool presented in [11] is used for the pantograph design and testing. In this tool, four classes of pantograph structures can be chosen to do the simulation, but the model of the contact line and the catenary is simpler than ours.

(ii) Reference [12] presents some ideas to simulate the pantograph-catenary interaction. In this work the state of the art is well defined, and the main concepts are described that should be present in a tool useful for engineers, design experts, and simulation experts.

(iii) Reference [13] has made a considerable effort in the solution of the pantograph-catenary dynamics. Some of this work has been taken into account in our work, but the solution proposed is limited to the mathematical model, and no considerations about the amount of time and space are taken into account to solve the problem in the proposed way. Moreover, there is no algorithm to be implemented in a tool suitable for engineers.

(iv) GA-Wire tool [14] is a commercial tool developed by GA-Energieanlagenbau Süd GmbH, which is a software to design overhead contact systems for the shortdistance traffic based on Auto-CAD. As a commercial software, without publishing the used algorithm, we do not know the main characteristics, but it only studies basic structures.

5.2. Interface. CALPE has been implemented using visual interface under Windows Platforms and Visual FoxPro () Microsoft) environment. It takes into account normal and stitched catenary, with one or two contact wires, several spans, overlapped spans (common area for two consecutive line spans), junction spans, and so forth.

CALPE tool, whose current user interfaces are in Spanish, and in process of translating into English, has a main window of control, in which it is possible to choose several options:

(i) selecting several utilities of habitual use, such as cut, copy, and exit, 


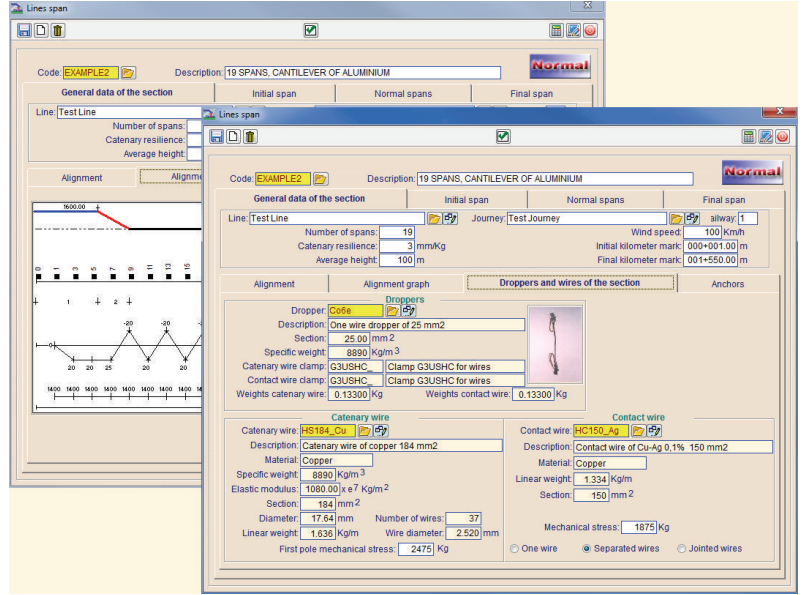

FIGURE 7: CALPE tool. Data input.

(ii) doing the main process, which allows the user to introduce and select the data and the execution of the analysis of the interaction,

(iii) the maintenance of the database system, designed with several files implementing the different tables of a relational database system following a previously designed entity-relation scheme: these tables implement the different auxiliary components that the users can use in the interaction: wires, droppers, pantographs, complements, and materials,

(iv) obtaining different reports about the auxiliary databases,

(v) some technical utilities for the correct work of the application.

The main procedure in the tool, called "Process," has been defined over a window interface. It allows the input of the different data types, selected among the previously introduced components in the data base systems, and even some other new data types.

In Figure 7, an example of data inputs over a window of the tool is presented. With the help of an optimal description for any calculation the user is able to locate it again. Further on its parameters can be modified for recomputation.

The design of the different windows in tabulated pages allows the user to manage the information intuitively and comfortably.

After the user has supplied all the data it is possible to execute the program. This process is short and depending on the complexity of the problem, it can spend some seconds, in general less than one minute. After that, the user can see, in a new window with tabulated pages, the different obtained results.

In Figure 8 we can see some examples of the main window of the results, either in tabulated or graphical form.

Finally, Figure 9 shows graphically the calculation of the data for cantilever, with the aid of the scheme of that component. A cantilever is a beam anchored at only one end. The beam carries the load to the support, where it is forced

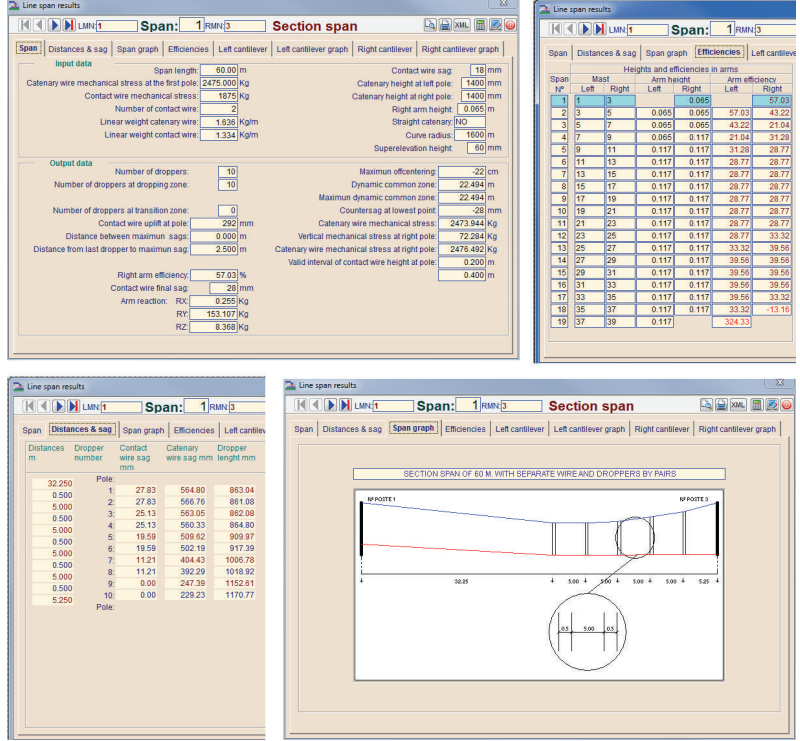

FIGURE 8: Presentation of results of spans.

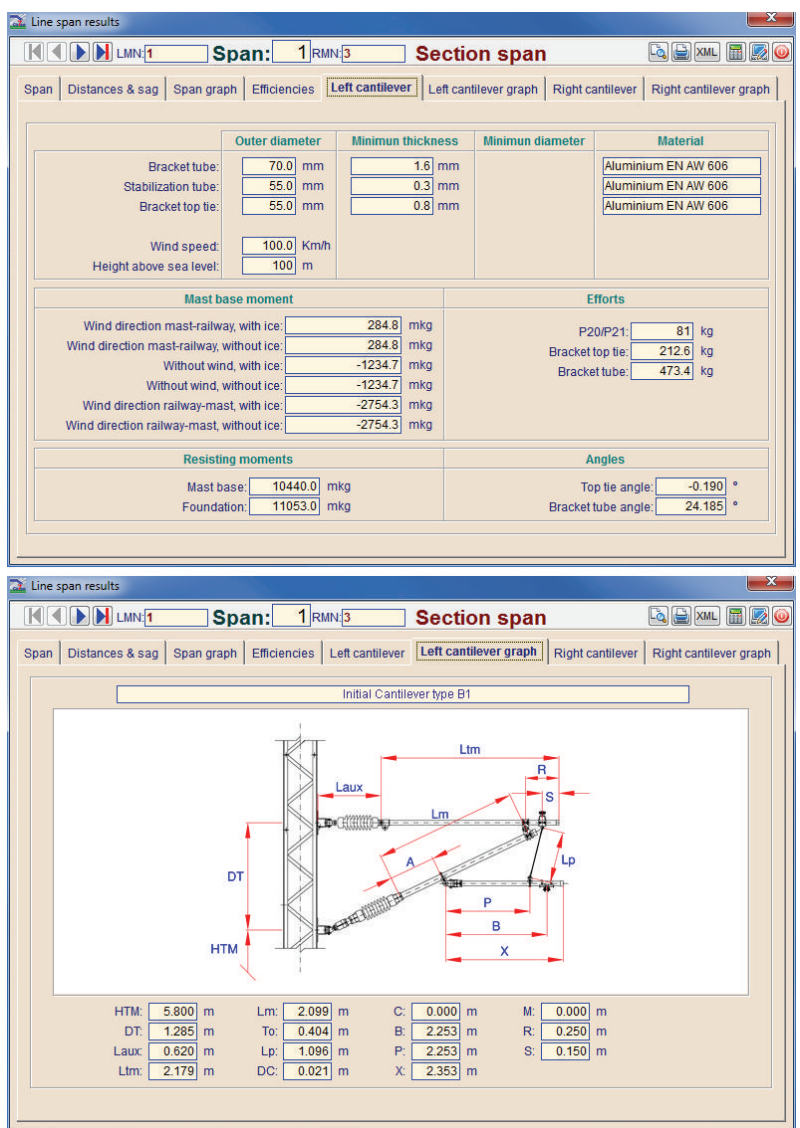

FIGURE 9: Presentation of results of cantilever.

against by a moment and shear force. The cantilever is an important part for overhanging structures. In this work, we are not presenting their calculation, but it is a part of the tool, and it is present in the database. 


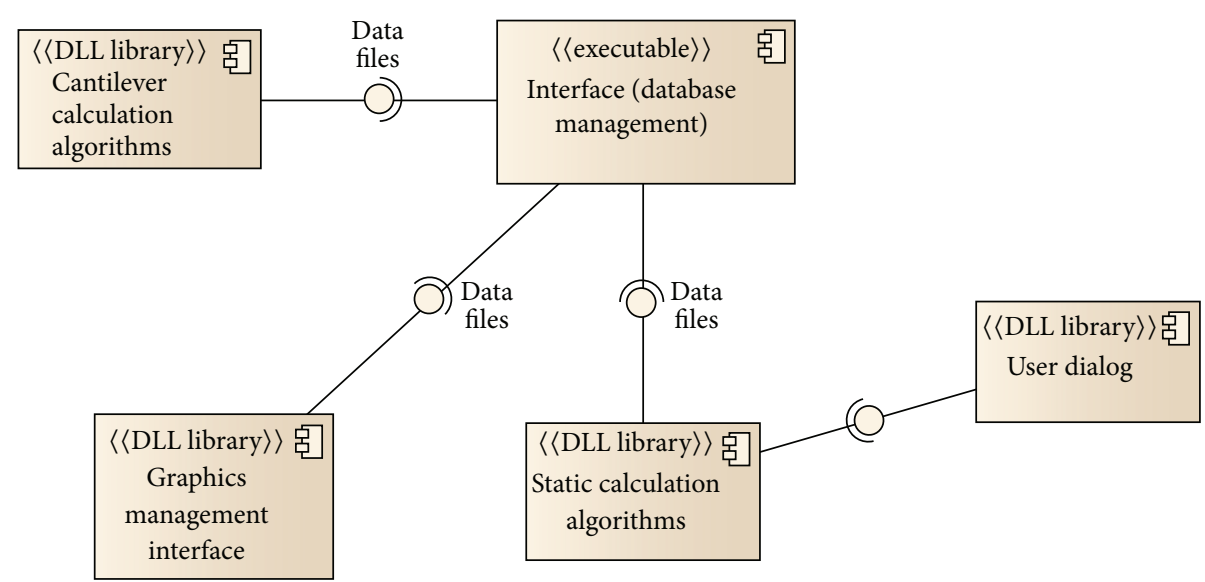

Figure 10: Diagram of components.

5.3. Design. After the definition of the tool, we design the application with the objective of creating a solution to a real and important problem for the industry. In order to do that, we have taken into account the different elements to obtain a tool suitable to be used by the engineer, the final user.

Thus, the different components of the tool, shown in Figure 10 by means of a UML (Unified Modeling Language) diagram, are as follows.

(i) The main program and the entry point to the tool manage the graphical user interface by means of a structure that organizes and facilitates the insertion of the large amount of information necessary for the calculation of the interaction in three dimensions. It is also responsible for the management of the relational database used to store auxiliary data needed for the calculations, and the database components used in the structure of the catenary. This main program has been written in Visual FoxPro.

(ii) The algorithms for the calculation of the span are written in standard $\mathrm{C}$ be using a DLL. This library contains the algorithms responsible for calculating the length of the droppers and other parameters describing the static structure of a line span. It obtains the information of the main program and returns the results of the calculation.

(iii) The graphical interface has been implemented using Visual $\mathrm{C}++$ and embedded into the user interface of the main application. It displays interactively (by selecting chart type, wire, collector shields, pantograph, zoom, etc.) the graphs generated from the results obtained from the library of interaction algorithms.

With respect to the database design, it consists of a relational database, where the information is stored, for later use in the calculations, and mainly to provide information about the components used in the assembly of the spans.
The design of the database has been made by means of two basic tables.

(i) Normal Span. It contains data about the span structure calculated in the interaction.

(ii) Cantilever. It contains the information that defines the structure of the cantilever that supports the line span cables.

Moreover, with respect to the table Normal Span, there are some other tables connected storing the description and technical characteristics of the components, which make up the structure of the span. Thus, we have wires, droppers, posts, and so one.

Also, the cantilever entity is associated with the entities normal span, initial span, and final span, which is connected with other entities storing the information related to straps, tubes, and support. On the other hand, the material entity is associated with all the entities due to the fact that it contains the material properties. We show the entity/relation diagram of the application in Figure 11.

5.4. Case Study. In this subsection, we present the design of a case study, based on a real design, with some variations in order to show the real power of the tool.

The overhead system we will use to illustrate our tool is based on a line span with 19 spans, and two contact wires along the line, with droppers located in pairs, because they are two contact wires supplying the electric energy to the pantograph.

All the spans, but the 12th, have the same length, of $60 \mathrm{~m}$. The first four of them are curved, and the rest in a line. This is because we assume that the span number 12th must overcome an overpass, so it is necessary to reduce the length of this span to $40 \mathrm{~m}$. and, progressively, beginning from the previous span, also reducing the height of the contact wire and the catenary, which also will be restored progressively in successive spans. Table 6 shows the length of spans and the catenary and contact wires height. 


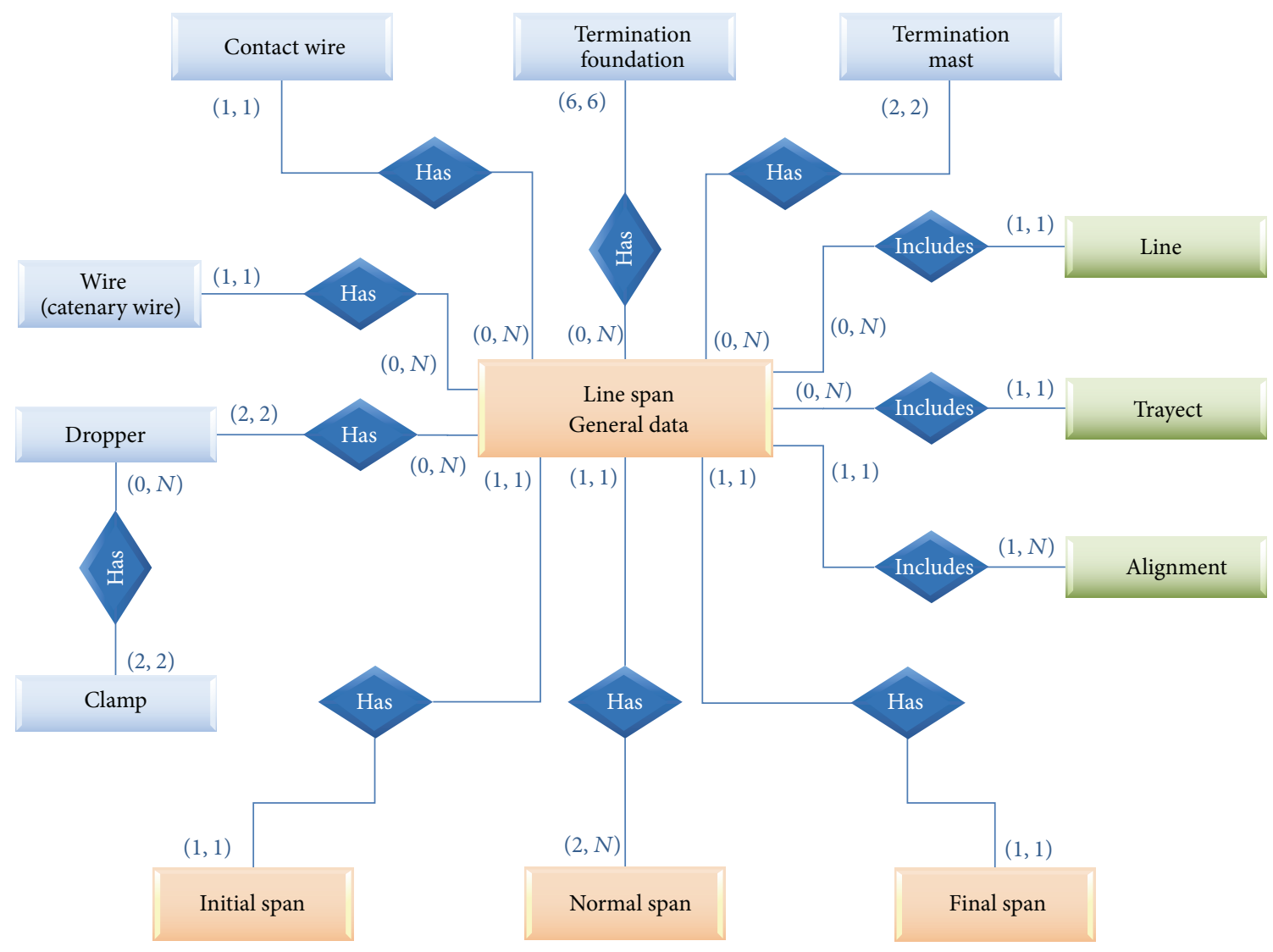

FIGURE 11: Diagram entity/relation.

TABle 6: Input data. Case study.

\begin{tabular}{lccccc}
\hline \multirow{2}{*}{ Span } & \multirow{2}{*}{ Length $(\mathrm{m})}$. & \multicolumn{2}{c}{ Catenary height } & \multicolumn{2}{c}{ Contact wire height } \\
& & Left & Right & Left & Right \\
\hline Init & 60 & 1400 & 1400 & 5300 & 5300 \\
$2-7$ & 60 & 1400 & 1400 & 5300 & 5300 \\
8 & 60 & 1400 & 1380 & 5300 & 5280 \\
9 & 60 & 1380 & 1360 & 5300 & 5300 \\
10 & 60 & 1360 & 1340 & 5280 & 5260 \\
11 & 60 & 1340 & 1320 & 5240 & 5220 \\
12 & 40 & 1320 & 1320 & 5220 & 5220 \\
13 & 60 & 1320 & 1340 & 5220 & 5240 \\
14 & 60 & 1340 & 1360 & 5240 & 5260 \\
15 & 60 & 1360 & 1380 & 5260 & 5280 \\
16 & 60 & 1400 & 1400 & 5300 & 5300 \\
\hline
\end{tabular}

Thus, we can summarize the characteristics of the catenary system that we will study in this example in the following:

(i) main characteristics of the spans: curve: spans 1 to 4 straight: spans 5-19;

(ii) distance 1st dropper to half-shaft: $5.25 \mathrm{~m}$.; (iii) distance between pairs of droppers: $5.00 \mathrm{~m}$.;

(iv) interior distance of the pair: $0.5 \mathrm{~m}$;

(v) length of span: $60 \mathrm{~m}$. with the exception of 12th with $40 \mathrm{~m}$.

In Figure 12 we show the alignment of the contact wire along the line span.

The execution time of the tool to solve this problem has been 15 milliseconds, using Intel Core Quad CPU Q9400 2,66 GHz under Windows 7 Operating System.

Once the problem is solved, the obtained results are shown in several tables that the user can select by using the interface of the application. The first one represents the efficiencies, which is shown in Figure 13.

Rest of figures show other results that we can obtain with the tool. We show only some of them corresponding to spans 1 and 12; the first one because it is the starting point of the calculation, and the 12th span, because it is the overpass point. Thus, Figures 14 and 17 show the main output data obtained, including efforts, reactions in the poles, mechanical stress, and some others, for this case.

Figures 15 and 18 show the geometry of each span, indicating the length of the respective droppers, and the sag along the contact and catenary wires.

Finally, Figures 16 and 19 show the graphics of the respective spans, the first one where it is located the section 


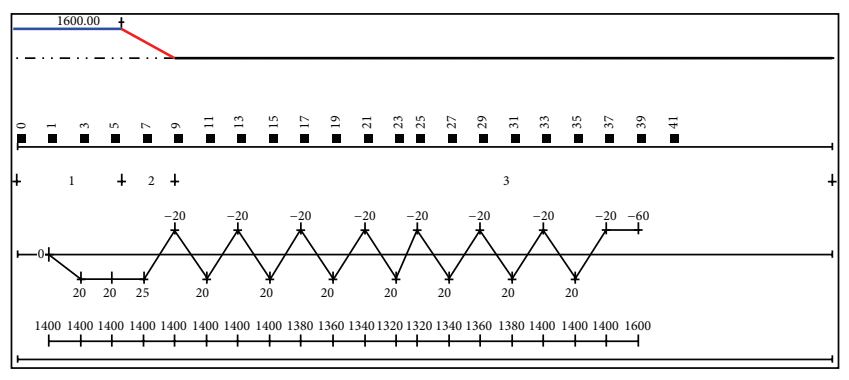

FIGURE 12: Alignment graph.

\begin{tabular}{|c|c|c|c|c|c|c|}
\hline \multirow{3}{*}{$\begin{array}{c}\text { Span } \\
\mathrm{N}^{\circ}\end{array}$} & \multicolumn{6}{|c|}{ Heights and efficiencies in arms } \\
\hline & \multicolumn{2}{|c|}{ Mast } & \multicolumn{2}{|c|}{ Arm height } & \multicolumn{2}{|c|}{ Arm efficiency } \\
\hline & Left & Right & Left & Right & Left & Right \\
\hline \begin{tabular}{|l|}
1 \\
\end{tabular} & 1 & 3 & & 0.065 & & 57.03 \\
\hline 2 & 3 & 5 & 0.065 & 0.065 & 57.03 & 43.22 \\
\hline 3 & 5 & 7 & 0.065 & 0.065 & 43.22 & 21.04 \\
\hline 4 & 7 & 9 & 0.065 & 0.117 & 21.04 & 31.28 \\
\hline 5 & 9 & 11 & 0.117 & 0.117 & 31.28 & 28.77 \\
\hline 6 & 11 & 13 & 0.117 & 0.117 & 28.77 & 28.77 \\
\hline 7 & 13 & 15 & 0.117 & 0.117 & 28.77 & 28.77 \\
\hline 8 & 15 & 17 & 0.117 & 0.117 & 28.77 & 28.77 \\
\hline 9 & 17 & 19 & 0.117 & 0.117 & 28.77 & 28.77 \\
\hline 10 & 19 & 21 & 0.117 & 0.117 & 28.77 & 28.77 \\
\hline 11 & 21 & 23 & 0.117 & 0.117 & 28.77 & 38.81 \\
\hline 12 & 23 & 25 & 0.117 & 0.117 & 38.81 & 44.94 \\
\hline 13 & 25 & 27 & 0.117 & 0.117 & 44.94 & 39.56 \\
\hline 14 & 27 & 29 & 0.117 & 0.117 & 39.56 & 39.56 \\
\hline 15 & 29 & 31 & 0.117 & 0.117 & 39.56 & 39.56 \\
\hline 16 & 31 & 33 & 0.117 & 0.117 & 39.56 & 39.56 \\
\hline 17 & 33 & 35 & 0.117 & 0.117 & 39.56 & 33.32 \\
\hline 18 & 35 & 37 & 0.117 & 0.117 & 33.32 & 10.08 \\
\hline 19 & 37 & 39 & 0.117 & & 10.08 & \\
\hline
\end{tabular}

FIgURE 13: Efficiencies.

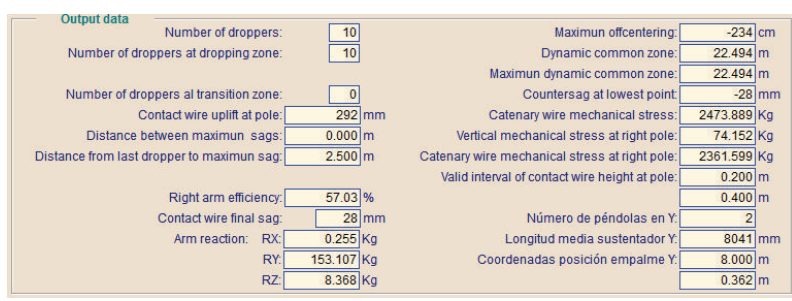

FIgURE 14: Span 1. Section span. Output data.

span, the first one of the line span, and the other one of the 12th span, which is located under the overpass.

This case study illustrates the power of the tool that allows for the engineer the design of a useful and efficient structure according to the demanded high standards, taking into consideration a very large set of possible restrictions presented in the real life.

\begin{tabular}{|c|c|c|c|c|}
\hline $\begin{array}{l}\text { Distances } \\
\mathrm{m}\end{array}$ & $\begin{array}{l}\text { Dropper } \\
\text { number }\end{array}$ & $\begin{array}{l}\text { Contact } \\
\text { wire sag } \\
\mathrm{mm}\end{array}$ & $\begin{array}{l}\text { Catenary } \\
\text { wire sag mm }\end{array}$ & $\begin{array}{l}\text { Dropper } \\
\text { lenght } \mathrm{mm}\end{array}$ \\
\hline 32250 & Pole: & & & \\
\hline 0.500 & 1: & 27.64 & 589.19 & 838.45 \\
\hline 5.000 & $2:$ & 27.64 & 591.65 & 835.99 \\
\hline 0.000 & $3:$ & 23.14 & 594.16 & 828.97 \\
\hline 0.500 & 4: & 23.14 & 592.20 & 830.94 \\
\hline 0.000 & 5: & 13.93 & 550.31 & 863.61 \\
\hline 0.500 & $6:$ & 13.93 & 543.90 & 870.02 \\
\hline 5.000 & $7:$ & 0.00 & 457.58 & 942.42 \\
\hline 0.500 & $8:$ & 0.00 & 445.44 & 954.56 \\
\hline 5.000 & $9:$ & 0.00 & 783.27 & 616.73 \\
\hline 0.500 & 10: & 0.00 & 832.91 & 567.09 \\
\hline 5.250 & Pole: & & & \\
\hline
\end{tabular}

Figure 15: Span 1. Section span. Droppers.

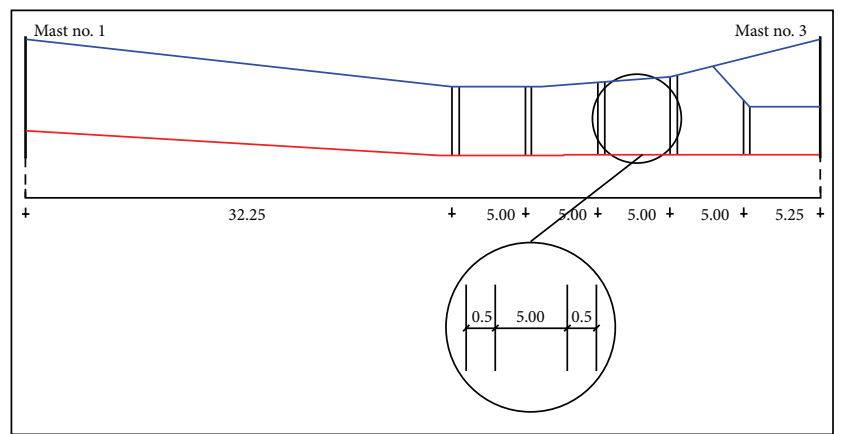

FIGURE 16: Span 1. Graph.

\section{Conclusions and Future Work}

In this work we have presented two methods for studying the problem of calculation of the forces actuating on catenary railways, considering two different hypotheses, in the first one considering the most accurate scenario where the cable weight is equivalent to a uniform load per unit length and in the second one considering the approximate load, assuming such weight as a uniform load per unit of horizontal length. The results have been contrasted with other examples published in the literature, obtaining a good numerical agreement, and with a nonexpensive cost in time of computing.

These methods have been used to develop a program written in Visual FoxPro and Visual $\mathrm{C}++$, the first one for the database management and the second for the calculation algorithms. As we have commented, this tool is not only an academic study, but it is currently used in the real industry. The public corporation ADIF is a company segregated from RENFE, the Spanish Railway Company, whose mission is the administration of railway infrastructures, and both companies are using the tool in the design of high speed railways (AVE program), recognizing that the tool is easy to use by noexpert users thanks to its graphical interface as well as in the introduction as the visualization of data. 


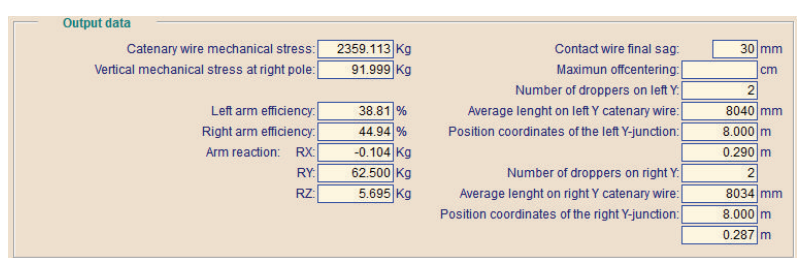

Figure 17: Span 12. Section span. Output data.

\begin{tabular}{|r|r|r|r|r|}
\hline $\begin{array}{l}\text { Distances } \\
\mathrm{m}\end{array}$ & $\begin{array}{l}\text { Dropper } \\
\text { number }\end{array}$ & $\begin{array}{l}\text { Contact } \\
\text { wire sag } \\
\mathrm{mm}\end{array}$ \\
\hline 5.250 & $\begin{array}{r}\text { Pole: } \\
\text { wire sag mm }\end{array}$ & $\begin{array}{l}\text { Catenary } \\
\text { wropher }\end{array}$ \\
\hline 0.500 & $1:$ & 0.00 & 750.96 & 569.04 \\
\hline 3.640 & $2:$ & 0.00 & 702.23 & 617.77 \\
\hline 0.500 & $3:$ & 0.00 & 321.34 & 998.66 \\
\hline 3.650 & $4:$ & 0.00 & 329.32 & 990.68 \\
\hline 0.500 & $5:$ & 19.20 & 360.39 & 978.81 \\
\hline 3.640 & $6:$ & 19.20 & 363.57 & 975.63 \\
\hline 0.500 & $7:$ & 28.80 & 378.87 & 969.93 \\
\hline 3.640 & $8:$ & 28.80 & 379.88 & 968.92 \\
\hline 0.500 & $9:$ & 28.80 & 379.42 & 969.38 \\
\hline 3.650 & $10:$ & 28.80 & 378.27 & 970.53 \\
\hline 0.500 & $11:$ & 19.20 & 362.05 & 977.15 \\
\hline 3.640 & $12:$ & 19.20 & 358.74 & 980.46 \\
\hline 0.500 & $13:$ & 0.00 & 326.74 & 993.26 \\
\hline 3.640 & $14:$ & 0.00 & 318.63 & 1001.37 \\
\hline 0.500 & $15:$ & 0.00 & 669.11 & 650.89 \\
\hline 5.250 & $16:$ & 0.00 & 714.47 & 605.53 \\
\hline & Pole: & & & \\
\hline
\end{tabular}

FIGURE 18: Span 12. Section span. Droppers.

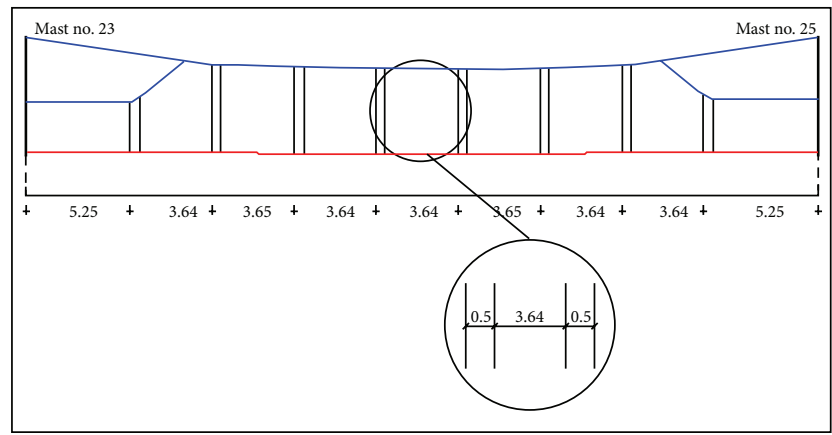

Figure 19: Span 12. Graph.

On the other hand, the continuous feedback of ADIF engineers allows us to improve the algorithms introduced in the tool, exploring new possibilities to incorporate in the tool.

Our proposed method allows us to calculate any type of catenary configuration and geometry and can be extended to the study of a system with stitched catenary. However, the calculation and subsequent assembly of this type of catenary present greater difficulty due to the existence of the false tensioner at the masts, with the aim to maintain a more uniform elasticity and a more favorable dynamic behavior. In most studies it is assumed that the central segment of the false tensioner is always horizontal, maintaining the symmetry of the span; however according to experience, this consideration is not completely true, because there is always some different slope for each mast, breaking the symmetry of the span, and also having a considerable influence in the configuration of the rest of the spans in the line. The study of this problem will be the subject of future work.

Also, the authors are now working on developing a model and extending the tool considering the rigid catenary with a range of different properties.

\section{Conflict of Interests}

The authors declare that there is no conflict of interests regarding the publication of this paper.

\section{Acknowledgment}

This work has been funded by the government of Castilla-La Mancha (Spain) under Project PII11-0198-3427.

\section{References}

[1] B. W. Kim, H. G. Sung, S. Y. Hong, and H. J. Jung, "Finite element nonlinear analysis for catenary structure considering elastic deformation," Computer Modeling in Engineering and Sciences, vol. 63, no. 1, pp. 29-45, 2010.

[2] M. Tur, E. García, L. Baeza, and F. J. Fuenmayor, "A 3D absolute nodal coordinate finite element model to compute the initial configuration of a railway catenary," Engineering Structures, vol. 71, no. 1, pp. 234-243, 2014.

[3] J. Benet, N. Cuartero, F. Cuartero, T. Rojo, P. Tendero, and E. Arias, "An advanced 3D-model for the study and simulation of the pantograph catenary system," Transportation Research Part C: Emerging Technologies, vol. 36, pp. 138-156, 2013.

[4] Y. H. Cho, K. Lee, Y. Park, B. Kang, and K.-N. Kim, "Influence of contact wire pre-sag on the dynamics of pantograph-railway catenary," International Journal of Mechanical Sciences, vol. 52, no. 11, pp. 1471-1490, 2010.

[5] G. Teichelmann, M. Schaub, and B. Simeon, "Modelling and simulation of railway cable systems," Journal of Applied Mathematics and Mechanics, vol. 85, no. 12, pp. 864-877, 2005.

[6] O. López-Garcia, A. Carnicero, and V. Torres, "Computation of the initial equilibrium of railway overheads based on the catenary equation," Engineering Structures, vol. 28, no. 10, pp. 1387-1394, 2006.

[7] F. P. Beer, E. R. Johnston, and E. R. Eisenberg, Vector Mechanics for Engineers, McGraw-Hill, 8th edition, 2007.

[8] M. Arnold and B. Simeon, "Pantograph and catenary dynamics: a benchmark problem and its numerical solution," Applied Numerical Mathematics, vol. 34, no. 4, pp. 345-362, 2000.

[9] Adif, "Dos aplicaciones de Adif ahorran en mantenimiento," Líneas Magazine, 2002 (Spanish), http://www.revistalineas .com/numero42/idi.htm.

[10] B. N. Datta, Numerical Linear Algebra and Applications, Brooks Cole, Pacific Grove, Calif, USA, 1984.

[11] A. Balestrino, O. Bruno, A. Landi, and L. Sani, "PANDA a friendly CAD tool for pantograph design and testing," in Proceedings of the 6th International Conference on Computer Aided Design, Manufacture and Operation in the Railway and 
Other Advanced Mass Transit Systems, pp. 817-826, Wessex IT, Lisbona, Portugal, September 1998.

[12] L. Drugge, T. Larsson, and A. Stensson, "Modelling and simulation of catenary-pantograph interaction," Vehicle System Dynamics, vol. 33, pp. 490-501, 2000.

[13] B. Simeon and M. Arnold, "Coupling DAES and PDES for simulating the interaction of pantograph and catenary," Mathematical and Computer Modelling of Dynamical Systems, vol. 6, no. 2, pp. 129-144, 2000.

[14] GA Wire, "GA-Energieanlagenbau Süd GmbH Technische Entwicklungen," Technical Developments Department, 2014, http://www.kummlermatter.nl/wp-content/uploads/GA-Wirebrochure2.pdf. 


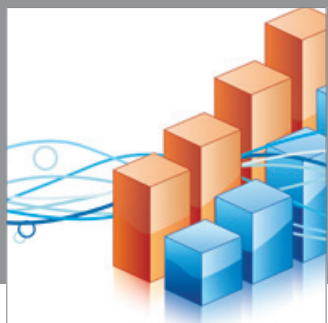

Advances in

Operations Research

mansans

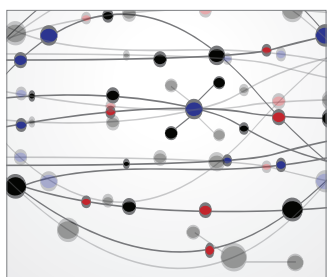

The Scientific World Journal
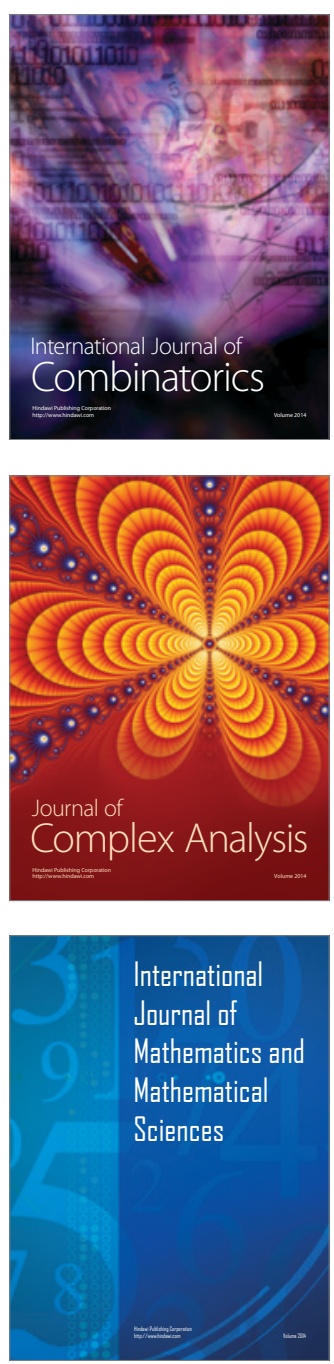
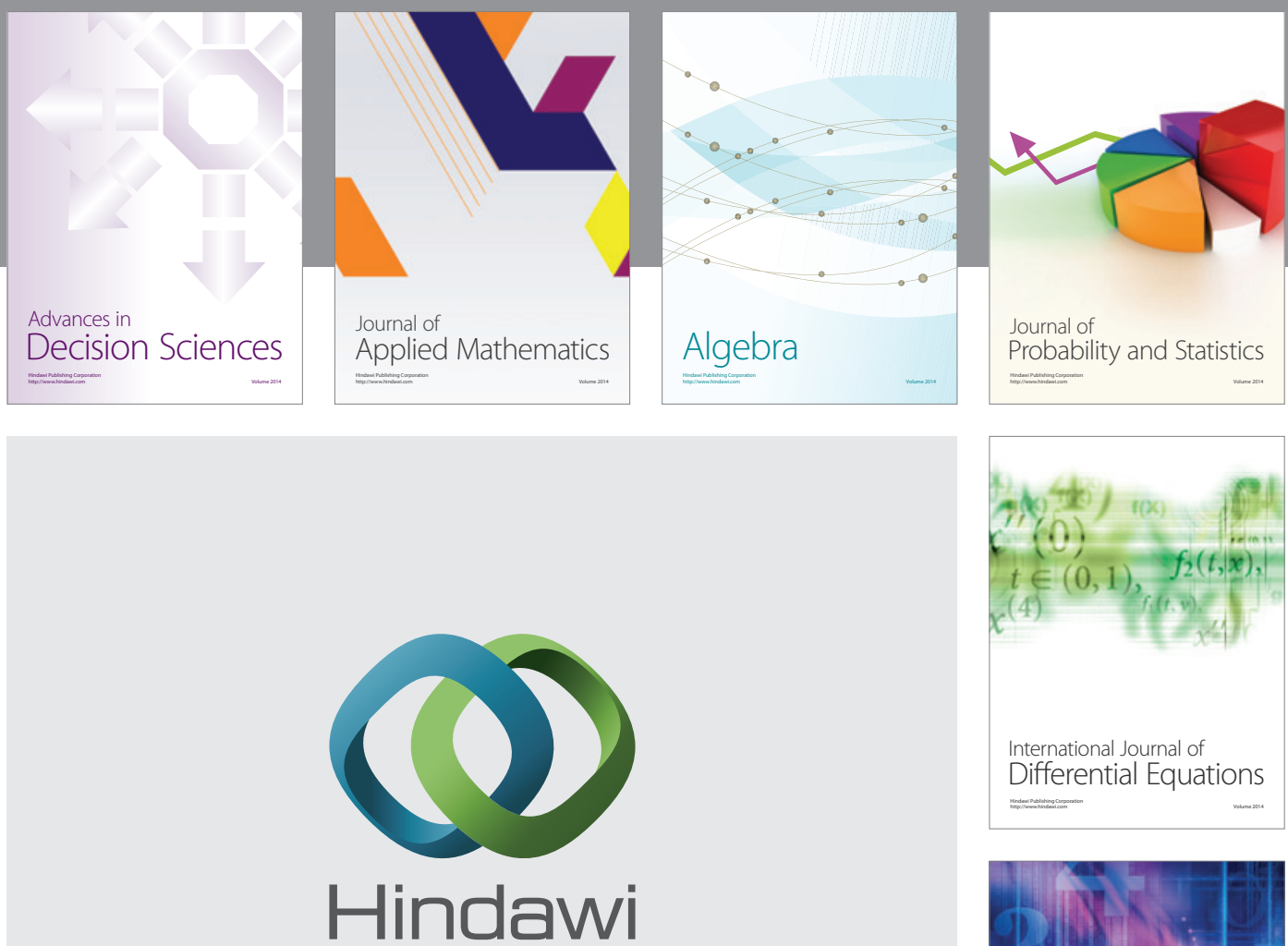

Submit your manuscripts at http://www.hindawi.com
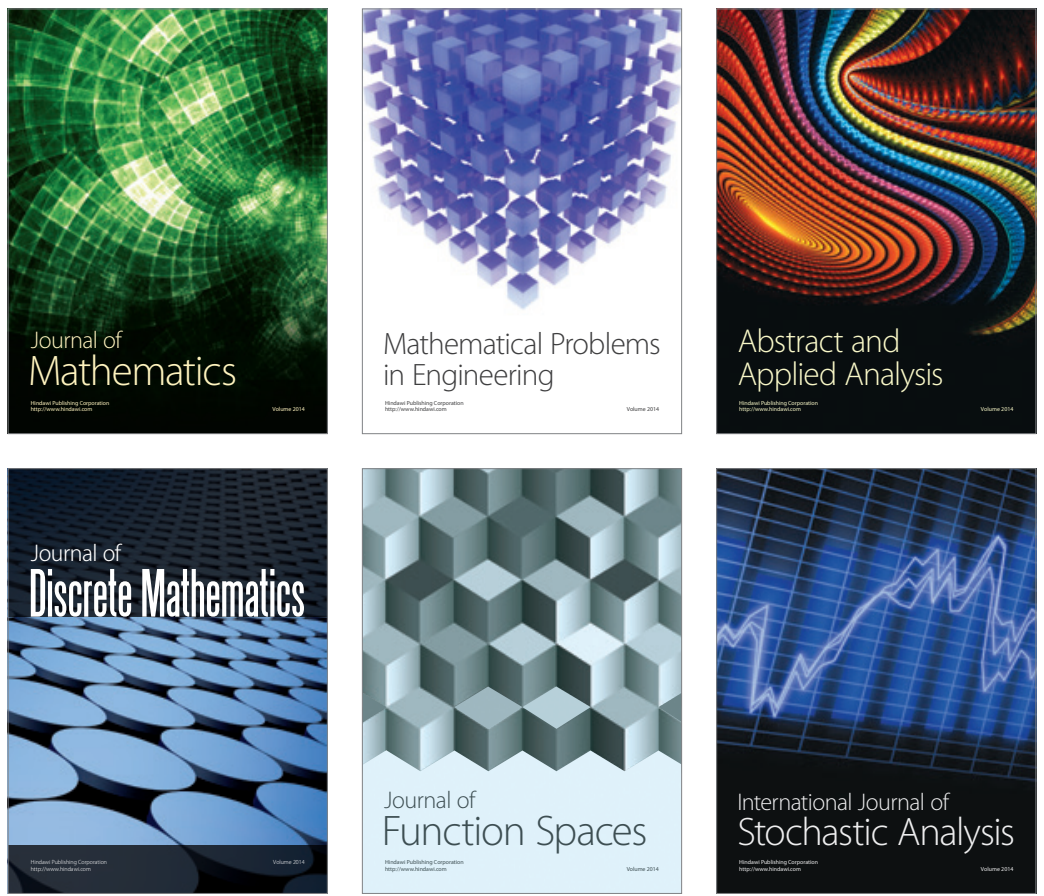

Journal of

Function Spaces

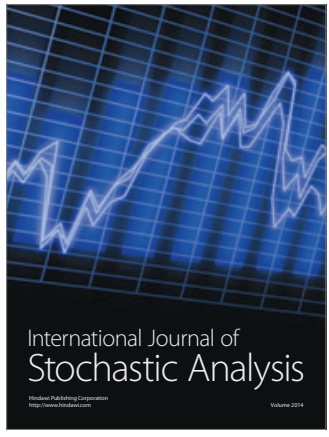

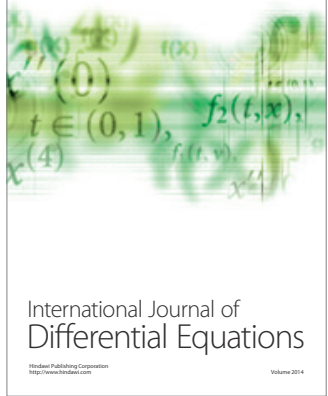
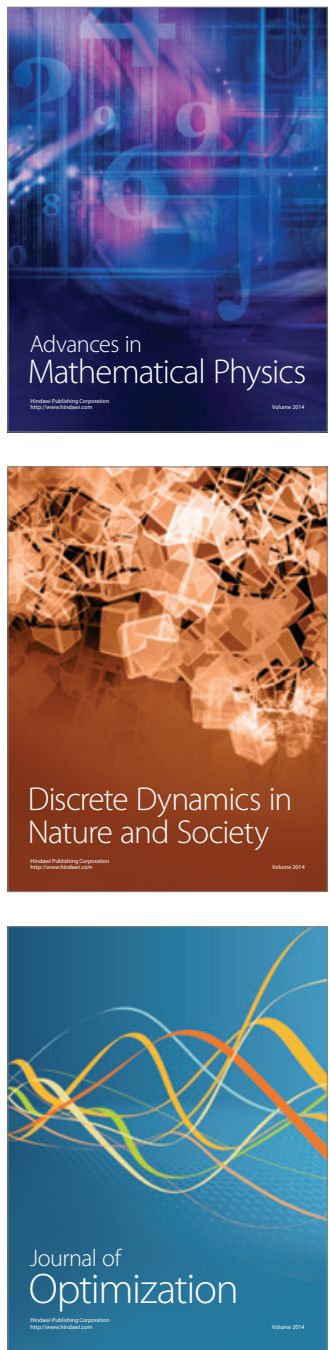